TU-438

June, 1993

\title{
The Weinberg Angle Without Grand Unification
}

\author{
T. Moroi, Hitoshi Murayama and T. Yanagida \\ Department of Physics, Tohoku University \\ Sendai, 980 Japan
}

\begin{abstract}
We assume that strong and electroweak interactions become strong at very high energies. With this assumption, we compute the low-energy gauge coupling constants $\alpha_{i}\left(m_{Z}\right)$ as a function of the cutoff scale, taking the supersymmetric standard model with $3+2 n$ families of quark and lepton multiplets. We find that only the five family case $(n=1)$ is consistent with the experimental values of the gauge coupling constants. This suggests the presence of a pair of families at $\sim 1 \mathrm{TeV}$.
\end{abstract}


Supersymmetry (SUSY) [1] has attracted many theorists in particle physics for a long time, since it is not only a mathematically consistent symmetry, but also it can eliminate all divergences more than quadratic in renormalizable quantum field theories. At present, the SUSY is expected to be a solution to the hierarchy problem (the presence of the light Higgs boson) [2]. However, in the SUSY extension of the standard electroweak theory with three families of quarks and leptons, the electroweak gauge interactions are not asymptotically free, and hence it may not be a consistent theory for all energies [3].

The grand unification (GUT) [4] of strong and electroweak interactions is certainly a possible solution to this problem, since all the gauge interactions become asymptotically free above the GUT scale. Furthermore, this SUSY-GUT [5] is strongly supported phenomenologically by the recent measurement of $\sin ^{2} \theta_{W}$ made at the LEP experiments [6]. The SUSY-GUT, however, has a serious problem to which no convincing solution has been found. Namely, the extreme fine tuning of parameters is required to produce a large mass splitting in Higgs multiplets. It is, therefore, very important to pursue alternative solutions to the problem of the non-asymptotically free nature of the electroweak interactions.

In the present paper we examine the proposal by Parisi [7], in which a physical cutoff $\Lambda$ is introduced such that the non-asymptotically free theory is valid up to the scale $\Lambda$. If the cutoff $\Lambda$ is sufficiently large, the gauge coupling constants at low energy (at the renormalization scale $\mu \sim 100 \mathrm{GeV}$ ) must be near the infrared stable fixed point $\alpha_{i}=0$. Moreover, if the coupling constants at $\Lambda$ are large enough, the low-energy coupling constants are approximately independent of the values of couplings at $\Lambda$ and are determined solely in terms of the cutoff $\Lambda$ and the number of matter multiplets [7]. Notably 30 years ago, Landau [8] suggested that the presence of a large number of fermions could give an explanation of the small value of the fine-structure constant $\alpha_{e m}=1 / 137$. A similar possibility has also been stressed based on a composite model of the gauge bosons [9]. 
In this paper we asuume that SUSY standard model with $3+2 n$ families of quarks and leptons. We take the cutoff scale $\Lambda$ as a free parameter which will be determined as a scale where all gauge coupling constants blow up. This is a crucial point to make our model viable phenomenologically, while in all previous analyses [10, 7, 8, 11] the cutoff $\Lambda$ is taken at the Planck scale, and hence they face a difficulty to obtain the correct value of the Weinberg angle [12].

To determine $\Lambda$ we compute the low-energy coupling constants $\alpha_{1}\left(m_{Z}\right), \alpha_{2}\left(m_{Z}\right)$ and $\alpha_{3}\left(m_{Z}\right)$ as a function of $\Lambda$ using the two-loop renormalization group (RG) equations [13]. We assume that the extra $2 n$ families form $n$ pairs of a family and a mirror family (family pairs) so that they can have $S U(2) \times U(1)$ invariant masses $m_{F}$. In this case, neutrinos in the extra families become massive Dirac fermions of the mass $m_{F}$ escaping the constraints from the LEP experiments. The results are shown in Fig. 1, for the number of family pairs $n=1,2,3$. In this Figure, we have taken $m_{F}=m_{S U S Y}=1 \mathrm{TeV}$ where $m_{S U S Y}$ is the SUSY-breaking scale. Note that $3+2 n=5$ is the minimum number of families which makes all the gauge coupling constants non-asymptotically free. To reproduce the correct values for the three low-energy gauge coupling constants, we find that the minimal number of the family pairs $n=1$ is the unique choice, and the cutoff turns out to be $\Lambda \simeq 2 \times 10^{16} \mathrm{GeV}$ [14]. Here we have taken $\alpha_{1}(\Lambda)=\alpha_{2}(\Lambda)=\alpha_{3}(\Lambda)=10$ as the initial value of the RG equations. We will restrict our discussions to the case $n=1$ hereafter.

To see that the low-energy gauge coupling constants are in fact independent of their values at the cutoff scale $\Lambda$, we vary the initial $\alpha_{i}$ between 10 and 100. Fig. 2 shows the running of the Weinberg angle $\sin ^{2} \theta_{W}$ just below the cutoff $\Lambda$. The initial values of $\sin ^{2} \theta_{W}$ are varied between 0.05 to 1.0 , and they rapidly approach the value $\sin ^{2} \theta_{W} \simeq 3 / 8$ around $10^{16} \mathrm{GeV}$. The further running down to the electroweak scale naturally reproduces the experimental value $\sin ^{2} \theta_{W}\left(m_{Z}\right)=0.2326 \pm 0.0008$, just as in the conventional SUSY-GUT. We have checked that even when $\alpha_{1}, \alpha_{2}$, and $\alpha_{3}$ are taken from 1 to 100 at the cutoff scale, their low-energy values scatter only within $2 \%$ for $\alpha_{1}\left(m_{Z}\right), 5 \%$ for 
$\alpha_{2}\left(m_{Z}\right)$ and $10 \%$ for $\alpha_{3}\left(m_{Z}\right)$. Thus, this scenario predicts all the low-energy gauge coupling constants almost irrespective of the initial values of the large gauge coupling constants at the cutoff scale.

The predicted gauge coupling constants are sensitive on the mass of the family pair as well as the SUSY-breaking scale, while insensitive on the initial gauge coupling constants. Varying $m_{F}=m_{S U S Y}$ between $100 \mathrm{GeV}$ and $10 \mathrm{TeV}$, we first determine the cutoff scale $\Lambda$ so that the correct weak-scale fine-structure constant $\alpha_{e m}^{-1}\left(m_{Z}\right)=127.9 \pm 0.2$ is obtained. With the determined $\Lambda$ we compute the Weinberg angle $\sin ^{2} \theta_{W}\left(m_{Z}\right)$ and the QCD running coupling $\alpha_{3}\left(m_{Z}\right)$ at the weak scale. The results are shown in Fig. 3. We see that the present model predicts one pair of a family and a mirror family at around $1 \mathrm{TeV}$ as well as the SUSY particles. Thus, the present model will be testable at the future supercollider experiments such as SSC or LHC 15.

A potential problem in the present model is the presence of the family pair at the $\mathrm{TeV}$ scale. Since they can have $S U(2) \times U(1)$ invariant masses, one needs to explain why they are so light compared with the cutoff scale $\Lambda \simeq 10^{16} \mathrm{GeV}$. A possible solution to this problem is given if they are Nambu-Goldstone supermultiplets arising from a spontaneous breakdown of some global symmetry $G$. An example is $E_{6} \rightarrow S O(10) \times$ $U(1)$, where the Nambu-Goldstone multiplets are $\mathbf{1 6}$ and $\overline{\mathbf{1 6}}$ of $S O(10)$ for the doubling realization case [16]. In this case the Nambu-Goldstone supermultiplets naturally have the masses of the order of the SUSY-breaking scale $m_{S U S Y}$ [17]. Thus, this example naturally explains the presence of a pair of a family and a mirror family at the SUSYbreaking scale.

The other problem is to clarify the physics at the cutoff scale. Since the cutoff $\Lambda$ is much lower than the Planck scale, it is plausible that the underlying physics is independent of the gravity. An intriguing possibility is that all the gauge fields of $S U(3) \times S U(2) \times U(1)$ are composite fields generated by the dynamics at the cutoff scale [18]. However, the dynamics to generate the composite gauge fields has not been yet clear to us. 


\section{References}

[1] D. Volkov and V.P. Akulov, JETP Lett. 16 (1972) 438;

J. Wess and B. Zumino, Nucl. Phys. B70 (1974) 39; Phys. Lett. 49B (1974) 52.

[2] M. Veltman, Acta Phys. Pol. B12, 437 (1981);

L. Maiani, Gif-sur-Yvette Summer School on Particle Physics, 11th, Gif-sur-Yvette, France, 1979 (Inst. Nat. Phys. Nucl. Phys. Particules, Paris, 1979);

S. Dimopoulos and S. Raby Nucl. Phys. B192, 353 (1981);

E. Witten, Nucl. Phys. B188, 513 (1981);

M. Dine, W. Fischler and M. Srednicki, Nucl. Phys. B189, 575 (1981).

[3] K. Wilson, Phys. Rev. D3 (1971) 1818.

[4] J.C. Pati and A. Salam, Phys. Rev. D10 (1974) 275;

H. Georgi and S. Glashow, Phys. Rev. Lett. 32 (1974) 438.

[5] E. Witten, in Ref. [2];

S. Dimopoulos, S. Raby, and F. Wilczek, Phys. Rev. D24, 1681 (1981);

S. Dimopoulos and H. Georgi, Nucl. Phys. B193, 150 (1981);

N. Sakai, Zeit. Phys. C11, 153 (1981).

[6] P. Langacker and M.-X. Luo, Phys. Rev. D44, 817 (1991);

U. Amaldi, W. de Boer and H. Fürstenau, Phys. Lett. 260B, 447 (1991);

W.J. Marciano, Brookhaven preprint, BNL-45999, April 1991.

[7] G. Parisi, Phys. Rev. D11 (1975) 909.

[8] L.D. Landau, A.A. Abrikosov, and I.M. Khalatnikov, Dokl. Akad. Nauk USSR 95 (1954) 773, 1177; ibid. 96 (1954) 261;

L.D. Landau and I. Pomeranchuk, Dokl. Akad. Nauk USSR 102 (1955) 489; 
L.D. Landau, "Niels Bohr and the development of physics," ed. W. Pauli (Pergamon Press, 1955).

[9] T. Saito and K. Shigemoto, Prog. Theor. Phys. 57 (1977) 242;

H. Terazawa, Y. Chikashige and K. Akama, Phys. Rev. D15 (1977) 480.

[10] L. Maiani, G. Parisi, and R. Petronzio, Nucl. Phys. B136 (1978) 115.

[11] H. Terazawa, Y. Chikashige, K. Akama and T. Matsuki, Phys. Rev. D15 (1977) 1181.

[12] The previous analyses [10, 0, 8, 9] did not include SUSY. To make all the three gauge coupling constants blow up at a single cutoff scale without SUSY, one has to introduce other matter multiplets in addition to the extra families in somewhat artificial manner; see, for example, H. Murayama and T. Yanagida, Mod. Phys. Lett. A7, (1992) 147.

[13] M.B. Einhorn and D.R.T. Jones, Nucl. Phys. B196, 475 (1982). We have chosen the normalization of the hypercharge coupling constant such that $\alpha_{1}=\frac{5}{3} \alpha_{2} \tan ^{2} \theta_{W}$.

[14] Since the gauge coupling constants become strong around the cutoff scale, the higher order effects may be important in determining the precise value of the cutoff scale $\Lambda$. However, the low-energy gauge coupling constants are insensitive on the detailed value of $\Lambda$.

[15] If we allow mass splitting in the family pair as well as in the SUSY spectrum, it is possible that some of the extra particles in the family pair are lighter than $1 \mathrm{TeV}$.

[16] In the doubling realization of $G$, there arise Nambu-Goldstone chiral multiplets $\Phi^{i}$ corresponding to each broken generators $T^{i}$. Since the scalar field of a chiral multiplet is a complex field, the number of the massless bosons are doubled. Half of them 
are true Nambu-Goldstone bosons and the other half are so-called quasi NambuGoldstone bosons. For details see W. Buchmüller, R. Peccei and T. Yanagida, Nucl. Phys. B227 (1983) 503.

[17] Once the SUSY is broken at $m_{S U S Y}$, the fermion fields of the Nambu-Goldstone multiplets acquire the masses of the order of $m_{S U S Y}$, since there is no protection from having the masses in general. On the other hand, the bosons get also the masses of the order of $m_{S U S Y}$ through the radiative corrections, since the gauge interactions break explicity the global symmetry $G$. See, W. Buchmüller, S. Love, R. Peccei, and T. Yanagida, Phys. Lett. 115B (1982) 233.

[18] T. Eguchi and H. Sugawara, Phys. Rev. D10 (1974) 4257. 


\section{Figure Caption}

Fig. 1 The cutoff dependence of the low-energy gauge coupling constants, $\alpha_{1}\left(m_{Z}\right)$ (Fig. 1a), $\alpha_{2}\left(m_{Z}\right)$ (Fig. 1b), and $\alpha_{3}\left(m_{Z}\right)$ (Fig. 1c), for various number of the family pairs $n=1,2,3$. The masses of the family pairs and SUSY-breaking scale are taken at $1 \mathrm{TeV}$. We adopt the gauge coupling constants given by P. Langacker, Pennsilvania University preprint, UPR-0492T, (1992).

Fig. 2 The running of the Weinberg angle $\sin ^{2} \theta_{W}$ below the cutoff $\Lambda=2 \times 10^{16} \mathrm{GeV}$. The gauge coupling constants at $\Lambda$ are taken randomly between 10 and 100 .

Fig. 3 The dependence of $\sin ^{2} \theta_{W}\left(m_{Z}\right)$ (Fig. 3a) and $\alpha_{3}\left(m_{Z}\right)$ (Fig. 3b) on the mass of the family pair $m_{F}$ and SUSY-breaking scale $m_{S U S Y}$ which are set equal. The cutoff $\Lambda$ is chosen to reproduce the correct weak-scale fine-structure constant $\alpha_{e m}\left(m_{Z}\right)=$ 127.9 . 